\title{
The Top Hat Electric Propulsion Plume Analyzer (TOPAZ): Preliminary Data on the BHT600 Cluster
}

\author{
Allen L. Victor ${ }^{*}$, Thomas H. Zurbuchen ${ }^{\dagger}$, and Alec D. Gallimore \\ Plasmadynamics and Electric Propulsion Laboratory \\ University of Michigan, Ann Arbor, MI, 48109 USA
}

\begin{abstract}
The testing of the Top Hat Electric Propulsion Plume Analyzer (TOPAZ) on the Busek BHT600 Hall thruster cluster is presented. TOPAZ incorporates a 'top hat' design with an analyzer constant of 100 resulting in a wide energy range and a high angular and energy resolution. An energy-image of the beam ions on the BHT600 Hall thruster cluster from 1.0 meters downstream is presented. Beam ions from each thruster are easily discerned, and seen to be focused from the centerline of each thruster. Measurements on a single thruster from a 60 degree plume angle are also made. Detected ions with energies significantly below the discharge voltage are believed to be created from either elastic collisions with low-energy particles or ionization near the cathode orifice. Future work will concentrate on the design of a time-of-flight analyzer for TOPAZ and further plume studies on the BHT600 cluster.
\end{abstract}

\section{Nomenclature}

$E \quad=$ energy of particle

$I \quad=$ measured current

$I_{n} \quad=$ normalized current

$K \quad=$ analyzer constant

$R_{l} \quad=$ deflection plate gap radius

$R_{2} \quad=$ grounded plate gap radius

$R_{3} \quad=$ top hat radius

$R_{C} \quad=$ gap centerline radius

$R_{G} \quad=$ guiding plate radius

$R_{P} \quad=$ particle radius of motion

$S \quad=$ aperture radius

$V_{D} \quad=$ deflection plate voltage

$p_{b} \quad=$ bass pressure

$p_{c} \quad=$ pressure corrected for xenon

$p_{i} \quad=$ indicated pressure

$q \quad=$ charge of particle

$\Delta R \quad=$ gap distance

$\alpha \quad=$ elevation angle

$\beta \quad=$ azimuthal angle of incoming particles

$\theta \quad=$ aperture angle

\section{Introduction}

$\mathrm{E}$ LECTRIC propulsion (EP) offers power-efficient, high specific impulse $\left(\mathrm{I}_{\mathrm{sp}}\right)$ options for deep-space missions as well as station keeping, orbital transfer, and attitude control requirements for near-Earth spacecraft. Hall thrusters are a type of EP system that utilizes electric and magnetic fields to produce thrust. Electrons emitted by a cathode travel upstream towards a positively-charged anode. A magnetic field that is applied in the perpendicular

\footnotetext{
* Graduate Student, Department of Aerospace Engineering, University of Michigan, Student Member AIAA

${ }^{\dagger}$ Research Scientist, Department of Atmospheric, Oceanic, and Space Sciences, University of Michigan

${ }^{*}$ Professor, Department of Aerospace Engineering, University of Michigan, Associate Fellow AIAA
} 
direction of the electric field hinders electron motion and creates a closed electron drift region. Propellant (e.g., xenon or krypton) is injected at the anode of an annular discharge channel, and ionized through collisions with the electrons caught in the closed electron drift region. The magnetic field has very little effect on the relatively massive ions. The electric field, however, accelerates the ions downstream away from the anode producing thrust. Currently, mid-power Hall thrusters achieve specific impulses between 1500-2500 seconds and electrical power efficiencies between 50-60\%. ${ }^{1}$

Recent trends in Hall thruster research by the USAF and US industry have included the high-power (>20 kW) regime. ${ }^{2}$ NASA is sponsoring high-power / high- $\mathrm{I}_{\text {sp }}(10 \mathrm{~kW} />=2000 \mathrm{~s})$ Hall thruster technology through the NASA Glenn Research Center (GRC). ${ }^{3}$ The NASA-457 Hall Thruster developed at GRC produced the highest power level and thrust $(75 \mathrm{~kW}$ and $2.9 \mathrm{~N})$ achieved by any xenon-propellant Hall Thruster. ${ }^{4}$ High $\mathrm{I}_{\mathrm{sp}}$ anode layer type (TAL) Hall thrusters have achieved specific impulses above $4100 \mathrm{~s}$ at this center as well. ${ }^{5}$

For these high-powered engines and future even more powerful engines being developed, plume characterization is imperative for determining their effect on spacecraft systems. Plasma transport properties, ionic charge state, and ion energy distributions are also important for understanding how Hall thrusters work and for improving their performance. ${ }^{6}$

One technique for determining the energy-to-charge distribution of plasma is to use an electrostatic analyzer. A specific geometry for the electrostatic analyzer, which allows for a wide field-of-view, is the top hat analyzer. This electrostatic analyzer consists of a sphere and a concentric shell with an aperture at the apex of the outer shell. The inner sphere is set to a specific voltage to allow for a narrow energy band of particles to pass through the aperture. By virtue of its geometry, the top hat analyzer is capable of having a 360-degree azimuthal field-of-view. Steering electric fields above the aperture allow for a field-of-view in the vertical direction as well. Structural constraints, however, diminish the total field-of-view in both directions.

The motivation for the design of the Top Hat Electric Propulsion Plume Analyzer (TOPAZ) is first discussed. The theory of operation for TOPAZ is described, and the relevant design parameters are listed. Measurements on the Busek BHT600 Hall thruster cluster conducted through TOPAZ are presented.

\section{Design Motivation}

Electrostatic analyzers have been and are currently employed on spacecraft to investigate space plasmas such as solar wind as well as the ionospheres and magnetospheres of Earth and other planets. ${ }^{7,8}$ Space plasmas offer a wide range of particle energies from less than $1 \mathrm{eV}$ to several MeV. This has led to design of electrostatic analyzers capable of detecting particles over several orders of magnitude in energy ${ }^{7}$; however, these types of plasmas have an ion number density several orders of magnitude lower than Hall thruster and ion engine plume.

Figure 1 describes the typical energy and number density ranges of space, laboratory, and Hall thruster and ion engine plasmas. The Hall thruster plume, the plasma of interest for TOPAZ, is nestled between laboratory plasmas (glow discharges and fusion experiments) and space plasmas (solar wind and the magnetotail) on the

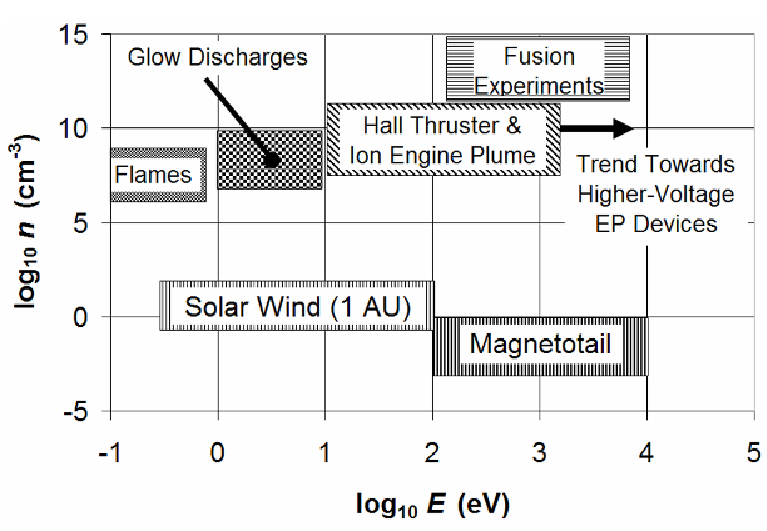

Figure 1. Number density and energy of typical space, laboratory, and electric propulsion plasmas. ${ }^{7,9,10}$ density scale. The energy range between Hall thruster plume and magnetotail plasma are similar. The primary difference between these two plasmas is the number density for the Hall thruster plume is several orders of magnitude greater.

There are many examples space plasma diagnostics through top hat analyzers. EP plume measurements with this type of device, however, are much rarer. The Plasma Experiment for Planetary Exploration (PEPE), flown on Deep Space 1 (DS1), included a dual top hat analyzer used to measure electrons and ions from the solar wind, spacecraft photoelectron sheath, and products of the xenon ion propulsion system. Low-energy xenon ions $(<40 \mathrm{eV}) \mathrm{created}$ from the beam ion interaction with neutral xenon particles were observed by PEPE. ${ }^{11}$

Although beam ions were not measured by PEPE due to the position and orientation of the thruster with respect to the instrument, the observation of charge-exchange ions provides evidence for the top hat analyzer as a plume diagnostics tool for measuring facility affects. Hall thrusters have been shown to yield higher current density profiles 
in the far-field plume at higher background pressures. It is theorized that charge-exchange ions created from beam ions and neutral background particles are the culprit for the elevated current densities. ${ }^{12}$ Diagnostic tools capable of characterizing the low energy charge-exchange ions as well as the high energy beam ions are therefore necessary to distinguish facility effects on plume diagnostics.

\section{Analyzer Design}

The TOPAZ design process is discussed in the following section. The design requirements for the analyzer are first described. A derivation of the ideal analyzer geometry is then presented. SIMION, an ion trajectory code, was used to predict the resolutions for TOPAZ. The final design for the analyzer was determined through an iterative process with SIMION.

\section{A. Design Requirements}

An increase in power is expected for EP thruster development, and the acceleration potentials (and hence beam ion energies) are expected to increase in the foreseeable future. While Hall thruster voltages are not expected to increase above $2 \mathrm{keV}$, ion thrusters that are being considered for deep-space missions may have xenon ion beams of energy above $6 \mathrm{keV}^{13}$. $\mathrm{A} \mathrm{X \textrm {Xe } ^ { 2 + }}$ ion would accelerate to $12-14 \mathrm{keV}$ at this potential; hence TOPAZ has been designed to have a high energy measurement capability up to $15 \mathrm{keV}$. The nature of a top hat analyzer allows for the lower bound to be close to $0 \mathrm{eV}$, since the plate potentials correspond directly with the measured energy. The lower energy bound therefore is set by the accuracy of the power supplies used.

Since TOPAZ is a far-field plume diagnostics instrument, an adequate field of view of the thruster is required to "image" the ions projected from the entire discharge channel. A $30^{\circ}$ vertical field-of-view allows for $57.7 \mathrm{~cm}$ of an object to be viewed from $1 \mathrm{~m}$ away, well within the size range of most thrusters. The azimuthal field-of-view is ideally $360^{\circ}$, but structural constraints diminish this to $112^{\circ}$. The angular resolutions of the field of view are within $2^{\circ} \times 2^{\circ}$ for the vertical and azimuthal directions, respectively. This resolution provides enough accuracy to determine a detected ion from the discharge channel, a different part of the thruster, or from the plume.

\section{B. Theory of Operation}

The top hat analyzer utilizes a radial electric field to guide ions through a spherical shell-shaped channel between a grounded plate and a negatively charged deflection plate. Figure 2 displays the key dimensions and plates for a top hat analyzer. The most important criterion is the ratio of the channel radius $R_{C}$ to the gap distance $\Delta R$ $\left(\Delta R=R_{2}-R_{I}\right)$ which sets the analyzer constant (Eq. 1).

$$
K \equiv \frac{R_{C}}{\Delta R}
$$

The analyzer constant $K$ determines the energy resolution, energy-to-voltage ratio, and other properties of the analyzer. The channel radius is simply the average of the inner and outer radii for the gap. Equating the applied force required to turn a particle at the channel radius with the electric field generated in the gap, the voltage is related to the energy-to-charge ratio. For high analyzer constants, the electric field can be assumed to be linear between the deflection plate and grounded plate. Equation (2) displays the simple relationship between the deflection plate voltage $V_{D}$, the analyzer constant, and the expected energyto-charge ratio to be measured.

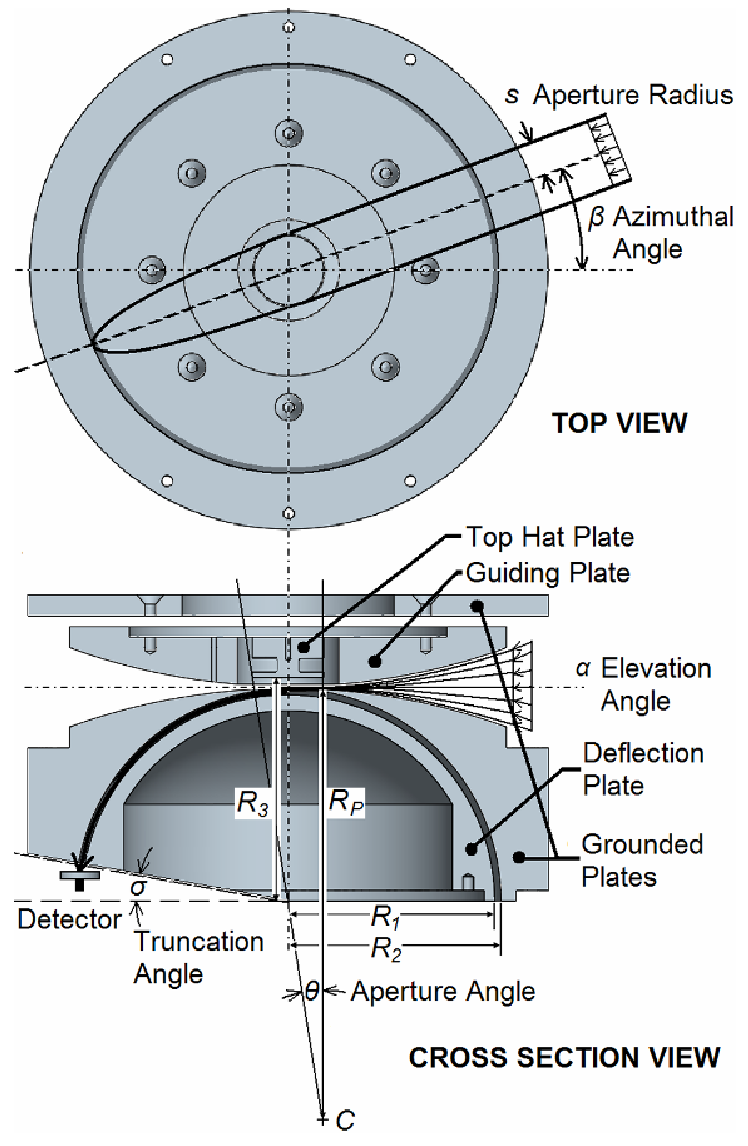

Figure 2. Principal design parameters of a top hat analyzer. ${ }^{12}$ 


$$
\frac{E}{q}=-\frac{K V_{D}}{2}
$$

The top hat radius $R_{3}$ and the aperture angle $\theta$ determine the average elevation angle and the effective aperture area for the measured ions, respectively. The maximum ratio of detectable ions to incoming ions is realized when the top hat radius $R_{3}$ is at least as large as the analyzer gap distance. ${ }^{12}$ This ratio yields a top hat radius of approximately $R_{3}=R_{1}+2 \Delta$.

The inner deflection plate radius $R_{l}$ is held to a negative plate potential to detect positively charged ions. The outer radius is kept at ground potential. The top hat plate is also usually held at ground, but can be biased to increase transmissivity if needed.

Guiding plates, which can vary either positively or negatively in plate potential, allow for variance in the vertical angular direction (elevation angle) for the measured ions. Ions coming from the selected elevation angle are guided into the top hat region such that their entrance angle is approximately horizontal above the deflection plate. The guiding plate radius of curvature is determined by setting the outer entrance angle of the surface slightly higher than the desired elevation angle $\alpha$ field-of-view. This angle is mirrored on the lower grounded plate surface. Through simple trigonometry the guiding plate radius $R_{G}$ is related to the top hat plate radius and the entrance surface angle in Eq. (3).

$$
R_{G}=\frac{R_{3}}{\sin \alpha}
$$

Particles enter the top hat aperture, and are turned at a radius $R_{P}$ due to the electric field generated between $R_{3}$ and $R_{1}$. Since $R_{3}-R_{1}=2 \Delta$, the electric field is half that of the gap, and $R_{P}$ is approximately $2 R_{2}$. The center of curvature for the particles is at point $C$ (see Fig. 2). To determine the optimum aperture angle $\theta$, the "grazing" trajectory of a particle is followed that touches the front lip of the top hat plate and follows the outer radius of the gap. The particle travels at a radius of $2 R_{2}$ due to the field generated between the top hat plate and the deflection plate. Through the construction of a right triangle between points $C$ and the top hat entrance lip and the outer gap radius, the optimum aperture angle $\theta$ can be derived (Eq. 4).

$$
\cos \theta=\frac{2 R_{2}-\Delta}{R_{P}}
$$

Since $R_{P} \approx 2 R_{2}$ and $R_{C} \approx R_{2}$ the aperture angle can be rewritten as a function of the analyzer constant through Eq. (1).

$$
\cos \theta \approx 1-\frac{1}{2 K}
$$

Through a two-term Taylor expansion of the cosine function, the aperture angle is directly correlated with the analyzer constant for $\theta<15^{\circ}$.

$$
\cos \theta=1-\frac{1}{2} \theta^{2} \ldots
$$

Therefore, the aperture angle (in radians) is proportional to the inverse square root of the analyzer constant.

$$
\theta \approx \sqrt{\frac{1}{K}}
$$


The optimum truncation angle $\sigma$ for ion focusing at the detector is $\sim \theta / 2 .{ }^{14}$ As the analyzer constant is increased above 50, this value becomes small and for TOPAZ is negligible and therefore is ignored. Ions entering over the aperture diameter $2 s$ are focused at the exit of the gap while maintaining their entrance azimuthal angle $\beta$ (shown in the top view of Fig. 2).

Only an approximate response can be estimated, since nonlinear surfaces and fringe effects from structural constraints are difficult to model analytically. SIMION, an ion optics program, allows for a more detailed design and characterization of TOPAZ to be determined.

\section{Analyzer Design through SIMION}

\begin{tabular}{|l|c|}
\hline \multicolumn{1}{|c|}{ PARAMETER } & VALUE \\
\hline Analyzer Constant, $K$ & 100 \\
\hline Inner Gap Radius, $R_{I}$ & $9.95 \mathrm{~cm}$ \\
\hline Outer Gap Radius, $R_{2}$ & $10.05 \mathrm{~cm}$ \\
\hline Gap Distance, $\Delta R$ & $1 \mathrm{~mm}$ \\
\hline Instrument Size $($ diameter $)$ & $24.6 \mathrm{~cm}$ \\
\hline Geometric Factor, $G(E)$ & $2.4 \times 10^{-5} \mathrm{~cm}^{2} \mathrm{sr} \mathrm{eV} / \mathrm{eV}$ \\
\hline Resolution $(\beta \times)$ & $2^{\circ} \times 2^{\circ}$ \\
\hline Field-of-View $\left(\Delta \beta \times \mathrm{x}^{\circ} \alpha\right)$ & $112^{\circ} \times 30^{\circ}$ \\
\hline Plate Material & Aluminum 6061 -T6 \\
\hline Insulator Material & Delrin $\AA$ and Glass Mica \\
\hline
\end{tabular}

Table 1. Physical characteristics of TOPAZ.

SIMION is a computer code that is capable of modeling ion optics problems with electrostatic and/or magnetic potential arrays. For the purposes of TOPAZ, only electrostatic fields were modeled. First, a model of TOPAZ was defined through a geometry file which included the volume definitions and potentials of the instrument. TOPAZ was assumed to be cylindrically symmetric through the eyes of SIMION. Then the electric potential $\varphi$ is solved for around the instrument through the Laplace equation. SIMION assumes a zero charge volume density (no space charge). ${ }^{15}$

After the potential field has been determined, ion trajectories are modeled by determining the electrostatic acceleration on the particle. SIMION incorporates a standard fourth order Runge-Kutta method for integrating out the ion's trajectory. ${ }^{15}$ The final design specifications and performance parameters are summarized in Table 1.

Experimental characterization and SIMION simulation of TOPAZ revealed the correlation between plate potentials, elevation angle $\alpha$, and ion energy-to-charge ratio E/q. Equations 8 and 9 display these relationships with the units in brackets.

$$
\begin{gathered}
V_{D}=-0.02083(E / q) \quad\left[\mathrm{eV} / \mathrm{e}_{\mathrm{c}}\right] \\
V_{G}=-\left(\frac{E / q}{262.4}\right)(\alpha[\mathrm{deg}]+1.871)
\end{gathered}
$$

SIMION is used to determine the transmissivity of TOPAZ. Since the transmissivity varies as a function of the elevation angle, the measured current is normalized across all elevation angles through equation 10 .

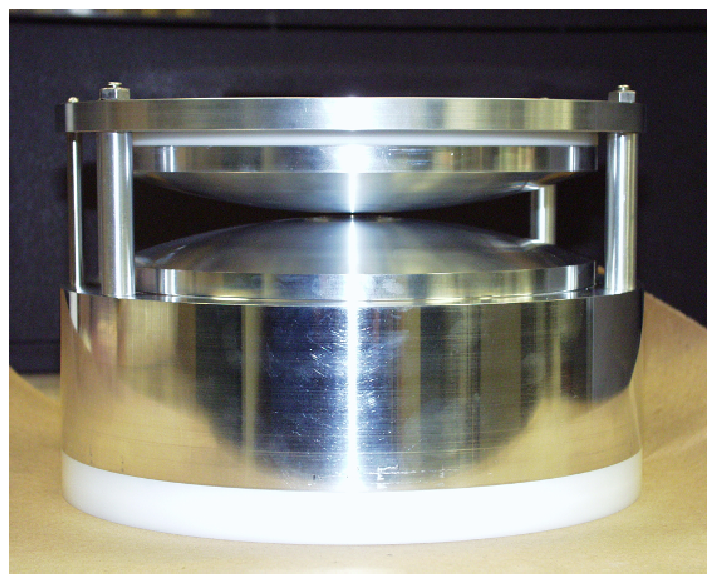

Figure 3. Final construction of TOPAZ.

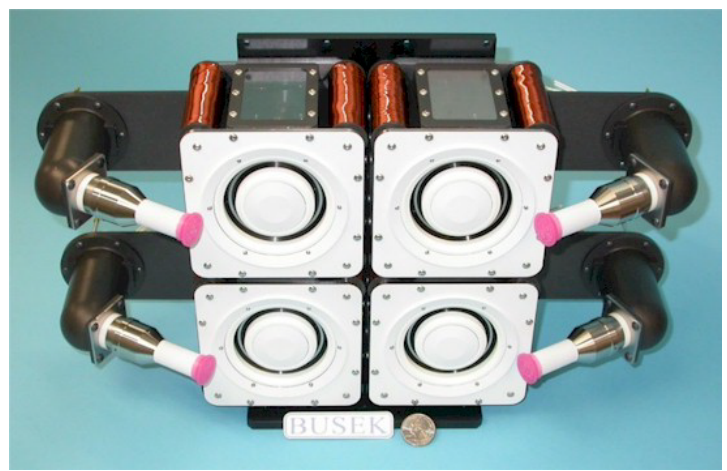

Figure 4. Cluster of four BHT600 Hall thrusters.

$$
I_{n}=\frac{I}{-\left(\frac{E / q}{241.4}\right)(\alpha[\mathrm{deg}]+1.871)+51.51}
$$


The relationship between transmissivity and elevation angle was determined through SIMION. The method of the design and characterization of TOPAZ is discussed in further detail in a previous article. ${ }^{16}$

\section{Measurements on the BHT600 Cluster}

To verify the operation of TOPAZ, as well as characterize the plume of a cluster of Hall thrusters, TOPAZ was placed one meter downstream of a cluster of four $600 \mathrm{~W}$ BHT600 Hall thrusters (Fig. 4). These thrusters operate on xenon propellant. Measurements were conducted at thruster plume angles of $0^{\circ}$ and $60^{\circ}$ (to the left of the cluster). Each Hall thruster was operated at $300 \mathrm{~V}$ and $2 \mathrm{~A}$.

\section{A. Experimental Setup}

All measurements were conducted in the University of Michigan's 6 m diameter by $9 \mathrm{~m}$ long Large Vacuum Test Facility (LVTF) at the Plasmadymamics and Electric Propulsion Laboratory (PEPL). Four model TM-1200 Re-Entrant Cryopumps are used to create an ultimate base pressure of $3 \times 10^{-7}$ Torr. The pressure for these measurements during cluster and single thruster operation were $5.0 \times 10^{-6}$ Torr and $1.96 \times 10^{-6}$ Torr, respectively. These values are corrected for xenon through equation $11 .^{17}$

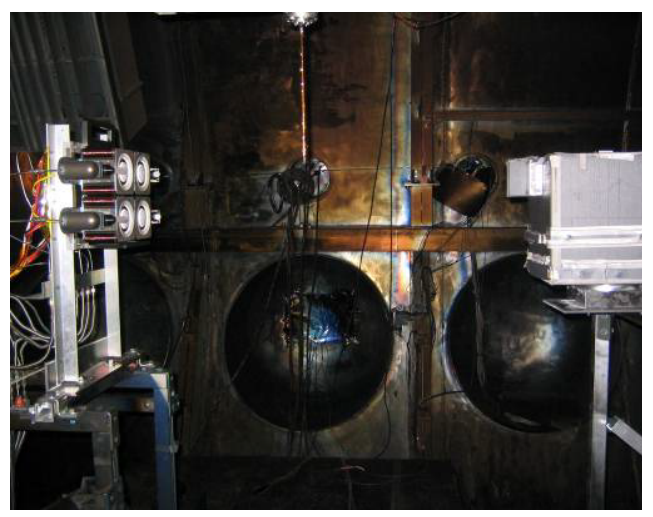

Figure 5. TOPAZ mounted one meter downstream of four BHT600 Hall thrusters inside the LVTF.

$$
p_{c}=\frac{p_{i}-p_{b}}{2.87}+p_{b}
$$

TOPAZ was placed exactly one meter downstream of the centerline of the four BHT600 Hall thrusters. A graphite box with a slit entrance is used to protect TOPAZ from the high energy beam flux. Figure 5 displays the BHT600 thrusters and TOPAZ inside the LVTF. The BHT600 cluster was acquired by PEPL for basic research on Hall thruster cluster characterization, their facility effects, and plume diagnostics. Each thruster has a $6 \mathrm{~cm}$ outside channel diameter and a centerline-to-centerline separation of $11 \mathrm{~cm}$ in the square-cluster configuration. Each thruster is operated at $300 \mathrm{~V}$ and $2 \mathrm{~A}$ with anode and cathode flow rates of $2.5 \mathrm{mg} / \mathrm{s}$ and $0.5 \mathrm{mg} / \mathrm{s}$, respectively. The cluster is mounted on two linear tables, which are positioned perpendicular to each other, allowing for two-dimensional movement. These tables allow for measurements by TOPAZ to be taken from different thruster angles.

A K\&M Electronics model 7550m channel electron multiplier (CEM) is employed to detect ions after they have passed through TOPAZ. The CEM operates by allowing ions to strike a ceramic surface with a high secondary electron emission rate. A voltage of $+1800 \mathrm{~V}$ is placed over a distance of $31.5 \mathrm{~mm}$ to induce secondary electron multiplication within the CEM, and the signal is amplified by $2 \times 10^{7}$. A Keithley picoammeter is used to measure the current generated by the CEM outside of the LVTF. To measure different azimuthal angles, TOPAZ and the CEM are rotated about the centerline of the analyzer by using a Daedal 20600RT rotary table. The rotational stage has a resolution of $0.001^{\circ}$ and is operated through LabView 6 software. Plate potentials were controlled by a Keithley 2410 SourceMeter and Kikusui power supplies.

A thermal couple was placed on TOPAZ to monitor the temperature of the instrument. During all tests the temperature remained between $20-55^{\circ} \mathrm{C}$.

\section{B. Experimental Results}

Data sweeps were taken by either varying the deflection plate voltage to generate energy profiles or by sweeping through elevation and azimuthal angles at constant plate voltages to piece together "images" of the thruster(s) at a particular energy. Figure 6 displays the energy-to-charge profile of the cluster down the centerline at a thruster angle of zero degrees. The peak energy-tocharge ratio is at $275 \mathrm{eV} / \mathrm{q}$.

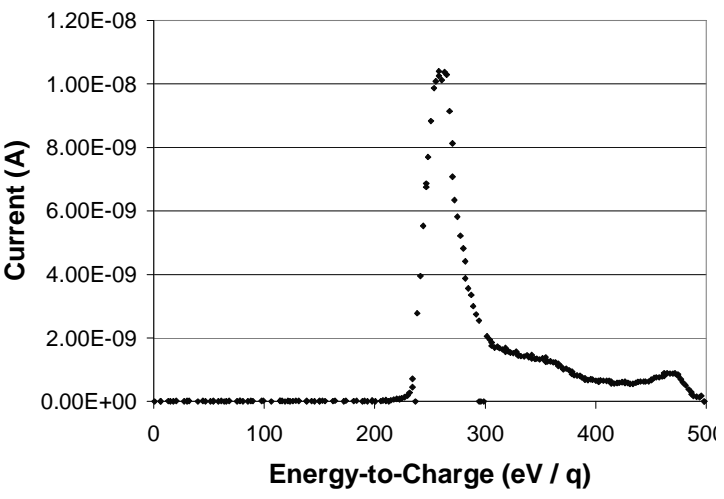

Figure 6. Energy profile of the BHT600 cluster. 


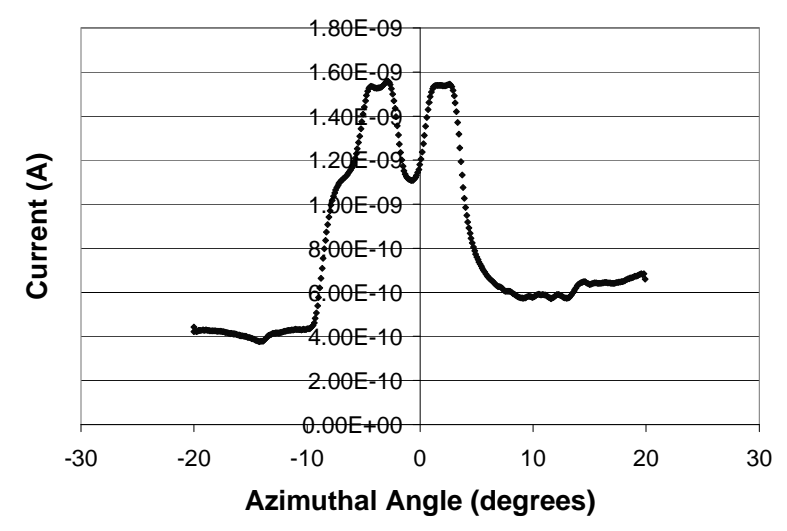

Figure 7. Azimuthal profile of $275 \mathrm{eV} / \mathrm{q}$ ions for the top two thrusters in the BHT600 cluster.

Figure 7 describes an azimuthal sweep of $275 \mathrm{eV} / \mathrm{q}$ ions across the top two thrusters of the cluster taken every $0.1^{\circ}$. To smooth the data without dismissing any trends beyond the azimuthal resolution ( 2 degrees) a five point boxcar average was performed. Two peaks emanating from the left and right centerlines of the cluster are seen. A noticeable dip in current exists between the thrusters. The separation distance of the peaks is $5.5^{\circ}$. A slight dip is also noticed at each peak, which may be indicative of the annular geometry of the Hall thruster.

Figure 8 displays an image of $275 \mathrm{eV} / \mathrm{q}$ ions is produced by combining azimuthal profiles with different guiding plate potentials (i.e., elevation angles $\alpha$ ) into a contour plot. Four peaks corresponding to each of the thrusters are readily identified. Although TOPAZ was level with the centerline of cluster, a large negative potential on the guiding plate was required to measure the beam ions. This corresponds with positive elevation angles. The image shift is believed to be caused by Debye shielding of the guiding plate at the entrance of TOPAZ. A similar effect was seen by Hofer with the Miniaturized Ion Energy Analyzer (MIEA) where the analyzer constant varied for different plume angles on the P5 Hall thruster. ${ }^{18}$ Since the guiding plate in TOPAZ contracts to within a few millimeters, it functions near the aperture, and the image form is maintained.

Measurements of a single BHT600 thruster were made on the bottom left thruster (approximately $-3^{\circ}$ in both the $\alpha$ - and $\beta$-directions). Energy-to-charge profiles were generated over a range of guiding plate potentials. By combining the profiles into a contour plot, the relationship between ion energy and elevation angle can be discerned. Figure 9 displays this contour plot. A positive trend is noticed between ion energy and elevation angle - ions emanating from below or in front of the thruster tend to have lower energies.

A similar contour plot of the energy-per-charge and azimuthal angle is displayed in Figure 10. Ions with energies below $275 \mathrm{eV} / \mathrm{q}$ tend to arrive from the left of the thruster near the cathode (see Figure 4 for cathode placement). Higher-energy ions are detected from the right side.

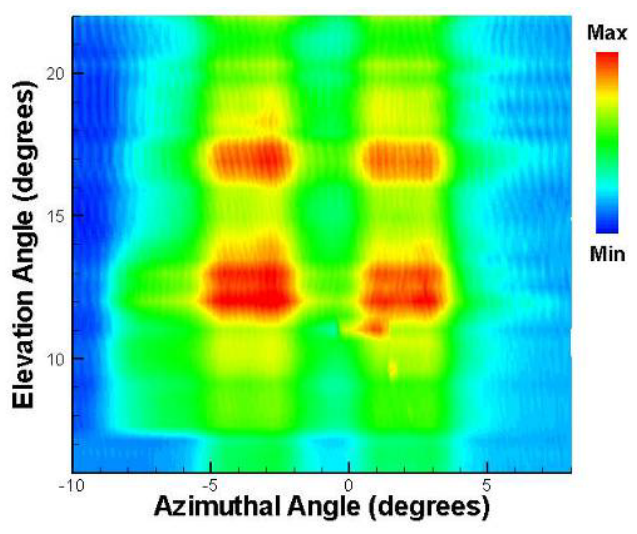

Figure 8. An image of $275 \mathrm{eV} / \mathrm{q}$ ions emanating from the BHT600 cluster.

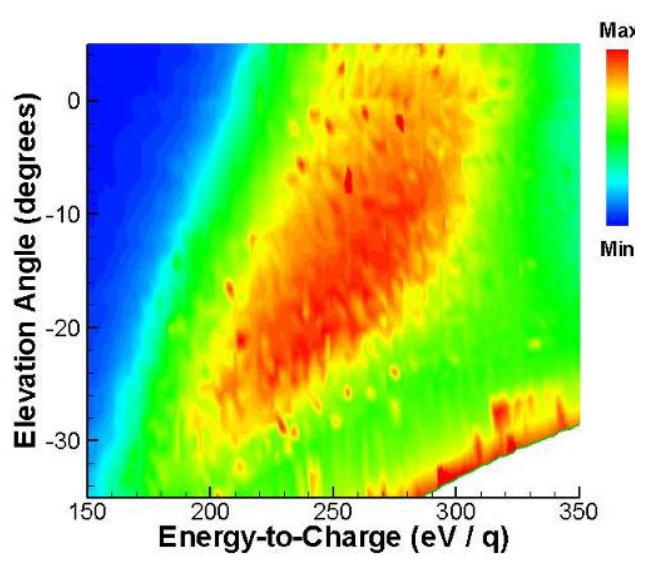

Figure 9. Elevation angle and energy-tocharge relationship for a single BHT600 thruster.

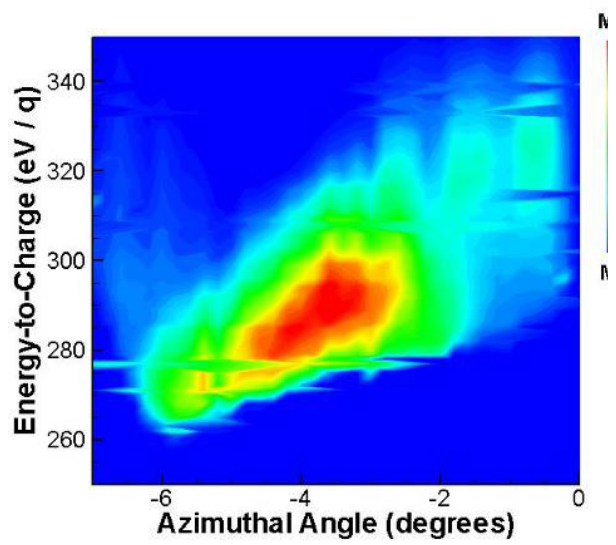

Figure 10. Azimuthal angle and energyto-charge relationship for a single BHT600 thruster. 


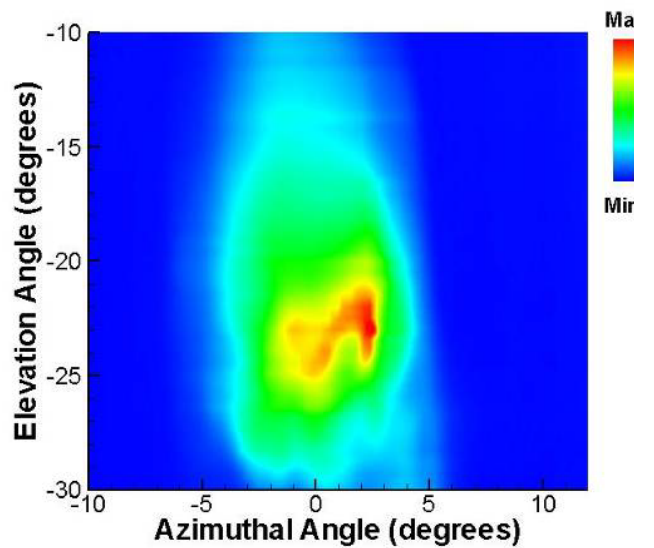

FIGURE 11. An image of $275 \mathrm{eV} / \mathrm{q}$ ions emanating from a single BHT600 thruster.
The single BHT600 thruster was positioned at a $60^{\circ}$ thruster angle with respect to TOPAZ. A similar image of $275 \mathrm{eV} / \mathrm{q}$ ions was measured for this thruster as was done with the cluster (Figure 11). Some discharge channel structure is present. The right side of the channel (side opposite of the cathode) displays a higher number of ions at $275 \mathrm{eV} / \mathrm{q}$, even though the left side is slightly closer and is less angled with respect to TOPAZ.

\section{Discussion}

Figure 6 describes the energy profile of the BHT600 cluster. Low-energy ions are not observed below the primary beam ion energy of $275 \mathrm{eV} / \mathrm{q}$. A possible reason for this is due to the very small angular acceptance for TOPAZ. In Figure 9, ions with energies below $275 \mathrm{eV} / \mathrm{q}$ are measured at lower elevation angles. The energy profile for the cluster is equivalent to a horizontal "slice" taken along $\alpha=-3^{\circ}$. At this angle for the single thruster, there are fewer ions with energies below $275 \mathrm{eV}$ as well. The data in both plots indicate that higher-energy ions tend to travel more in line with the thruster centerline, while lower-energy ions diverge at larger elevation angles. Figure 10 indicates these low-energy ions are detected from the left of the thruster near the cathode as well.

Neutral particles and low-energy ions emitted by the cathode could decrease beam ion energy through elastic collisions. Since the cathode plume particles are released in front of the thruster and are heavily concentrated on the left of the thruster (near the cathode orifice), elastic and charge exchange collisions are more likely to occur in the lower-left area of the thrusters from the viewpoint of TOPAZ. In addition, ions that are created near the cathode and outside the discharge channel will have lower acceleration potentials and hence lower energies. The electron stream from the cathode to the anode could ionize neutral particles that accelerate only through part of the potential drop generated by the anode.

Figures 7 and 8 describe where the beam ions of $275 \mathrm{eV} / \mathrm{q}$ are detected from the cluster. The two peaks in the profile of Fig. 7 are spaced $5.54^{\circ}$ apart. The distance from the cluster to the aperture in TOPAZ is $112.3 \mathrm{~cm}$. Since the thrusters are spaced $11 \mathrm{~cm}$ apart, they have an apparent azimuthal angular spacing of $5.59^{\circ}$ with respect to TOPAZ. The matching of the measured peak currents and the angular spacing indicate the ion production/acceleration is most likely concentrated around the centerline of each thruster. The width of the peaks is approximately $2^{\circ}$. This corresponds to a distance of $3.9 \mathrm{~cm}$ at the BHT600 cluster. Since the outside diameter of the discharge channel is $6 \mathrm{~cm}$, it is likely that the ions are most likely being measured from within this diameter. The image of $275 \mathrm{eV} / \mathrm{q}$ ions from the cluster indicates the beam ions from each thruster are not "blurred" together. Their trajectory is maintained into the far-field plume and beam ions from each thruster are easily discerned.

Figure 11 displays a beam ion image of a single thruster at a $60^{\circ}$ plume angle. The oval shape of the image corresponds with the angle of the thruster with respect to TOPAZ. A higher current is detected from the right side of the thruster, even though it is slightly further and at a higher angle of incidence. However, as stated previously the cathode interaction with the plume could decrease the energy of beam ions on the left side of the thruster near the cathode orifice. These ions would not be detected in the $275 \mathrm{eV} / \mathrm{q}$ image of the thruster.

\section{Conclusions}

Measurements on the Busek BHT600 Hall thruster cluster were conducted through TOPAZ. Energy profiles depicting ion energies near the discharge voltage of the thrusters verify the instrument's utility for Hall thruster cluster plume characterization. Beam ions along the centerline of each thruster have energies closest to the discharge voltage. Ions emanating from near the cathode tend to have energies significantly lower than the discharge voltage. Either elastic and/or charge exchange collisions with low-energy particles or ion production at potentials lower than the anode potential are conjectured to be possible causes for this phenomenon. This relationship could also affect measurements of beam ions at off axis plume angles as well.

\section{Future Work}

Further measurements on the BHT600 Cluster (such as imaging the cluster at different plume angles and energies) will be performed. A time-of-flight (TOF) analyzer is being developed for TOPAZ to measure the massper-charge of the particles. An ion beam will be employed to characterize the TOF operation. A partial velocity

8

American Institute of Aeronautics and Astronautics 
distribution function (within the field-of-view) can be directly measured by correlating the energy- and mass-percharge profiles. This will be compared with existing laser-induced fluorescence (LIF) measurements to verify the instruments viability as a novel and useful tool for conducting detailed far-field plume diagnostics on EP devices.

\section{Acknowledgments}

The authors greatly acknowledge Dr. Patrick Koehn for his endless support and lease of the ion accelerator, channeltron, and vacuum chamber for the characterization of TOPAZ. The authors thank Jessica Brooks for her SIMION simulation support and Chuck Navarre and Robb Gillespie for their highly precise machining of the parts. The authors thank Robert Lobbia for the setup and operation of the Busek BHT600 Hall thruster cluster. Finally, the authors thank Robert Lundgren for offering his expertise on the design of TOPAZ. A. Victor is supported by the NASA-funded Michigan Space Grant Consortium as well as the Department of Aerospace Engineering at the University of Michigan.

\section{References}

${ }^{1}$ Haas, J. M., Gulczinski, F. S., and Gallimore, A. D., "Performance Characteristics of a 5 kW Laboratory Hall Thruster," AIAA-97-3503, 34 ${ }^{\text {th }}$ Joint Propulsion Conference, Cleveland, OH, July 1998.

${ }^{2}$ Spores, R. A., Spanjers, G. G., Birkan, M., Lawrence, T. J., "Overview of the USAF Electric Propulsion Program," AIAA2001-3225, 37 $7^{\text {th }}$ Joint Propulsion Conference, Salt Lake City, UT, July, 2001.

${ }^{3}$ Dunning, J., Sankovic, J., "NASA's Electric Propulsion Program," AIAA-2000-3145, 36 ${ }^{\text {th }}$ Joint Propulsion Conference, Huntsville, AL, July 2000.

${ }^{4}$ Manzella, D., Jankovsky, R., Hofer, R., "Laboratory Model 50 kW Hall Thruster," AIAA-2002-3676, 38 ${ }^{\text {th }}$ Joint Propulsion, Indianapolis, IN, July 2002.

${ }^{5}$ Jacobson, D. T., Jankovsky, R.S., Rawlin, V. K., Manzella, D. H., "High Voltage TAL Performance," AIAA-2001-3777, $37^{\text {th }}$ Joint Propulsion Conference, Salt Lake City, UT, July 2001.

${ }^{6}$ Gallimore, A. D., "Near- and Far-Field Characterization of Stationary Plasma Thruster Plumes," Journal of Spacecraft and Rockets, Vol. 38, No.3, 2001, p. 441-453.

${ }^{7}$ Bame, S. J., McComas, D. J., Young, D. T., Belian, R. D., "Diagnostics of Space Plasmas," Review of Scientific Instruments, Vol. 57, No. 8, August 1986, p. 1711-1716.

${ }^{8}$ Vilppola, J. H., Tanskanen, P. J., Huomo, H., Barraclough, B. L., "Simulations of the Response Function of a Plasma Ion Beam Spectrometer for the Cassini Mission to Saturn," Review of Scientific Instruments, Vol 67, No. 4, April 1996, pp. 14941501.

${ }^{9}$ Hofer, R. R., Haas, J. M., Gallimore, A. D., "Ion Voltage Diagnostics in the Far-Field Plume of a High-Specific Impulse Hall Thruster," AIAA-2003-4556, 39th Joint Propulsion Conference, Huntsville, AL, July 20-23, 2003.

${ }^{10}$ Herman, D. A., Gallimore, A. D., "Comparison of Discharge Plasma Parameters in a 30-cm NSTAR Type Ion Engine with and without Beam Extraction," AIAA-2003-5162, 39th Joint Propulsion Conference, Huntsville, AL, July 20-23, 2003.

${ }^{11}$ Young, D. T., Nordholt, J. E., Hanley, J. J., "Plasma Experiment for Planetary Exploration (PEPE) DS1 Technology Validation Report," JPL Publication 00-10, 2000.

${ }^{12}$ Walker, M. L. R., Hofer, R. R., Gallimore, A. D., "The Effects of Nude Faraday Probe Design and Vacuum Facility Backpressure on the Measured Ion Current Density Profile of Hall Thruster Plumes," AIAA-2002-4253, 38th Joint Propulsion Conference, Indianapolis, IN, July 7-10, 2002.

${ }^{13}$ S. Oleson, "Electric Propulsion for Project Prometheus,"AIAA-2003-5279, 39th AIAA/ASME/SAE/ASEE Joint Propulsion Conference and Exhibit, Huntsville, Alabama, July 20-23, 2003.

${ }^{14}$ Carlson, C. W., McFadden, J. P., "Design and Application of Imaging Plasma Instruments," Measurements Techniques in Space Plasmas: Particles, edited by R. F. Pfaff, J. E. Borovsky, and D. S. Young, AGU Geophysical monograph, Washington, DC, 1998, pp. 125-140.

${ }^{15}$ Dahl, D. A., SIMION 3D Version 7.0 User's Manual, Idaho National Engineering and Environmental Laboratory, Idaho Falls, ID, 2000, pp. 2-5, E-12.

${ }^{16}$ Victor, A. L., Zurbuchen, T. H., Gallimore, A. D., "Development of the Top Hat Electric Propulsion Plume Analyzer (TOPAZ)," AIAA-2004-4099, 40th Joint Propulsion Conference, Fort Lauderdale, FL, July 11-14, 2004.

${ }^{17}$ Dushman, S., Scientific Foundations of Vacuum Technique, Vol. 4, Wiley, N.Y., 1958.

${ }^{18}$ Hofer, R. R., Haas, J. M., and Gallimore, A. D., "Development of a 45-Degree Parallel-Plate Electrostatic Energy Analyzer for Hall Thruster Plume Studies: Preliminary Data," IEPC-99-113, 26th International Electric Propulsion Conference, Kitakyushu, Japan, October 1999. 